\title{
Validation Data for Aircraft Noise Shielding Prediction
}

\author{
Karl-Stéphane Rossignol · Jan Werner \\ Delfs • Michael Mößner • Markus Lummer • \\ Jianping Yin
}

the date of receipt and acceptance should be inserted later

\begin{abstract}
The design of future low-noise aircraft will require an account of noise shielding as a fundamental design parameter. This aspect was already recognized in the seventies, where early experimental work set the ground on possible solutions to the conventional, short and reduced take-off and landing noise problem. Current lownoise aircraft design concepts of the hybrid-wing-body type require establishing a new knowledge base to allow an assessment of their potential noise benefit. Experimental work done in the course of the last 15 years, slowly moved in this direction with the establishment of new testing methodologies. Currently available databases render an estimation of the turbomachinery noise shielding benefit of specific design choices on aircraft of the hybrid-wing-body type possible. Still, research in this domain would strongly benefit from the development of reliable and flexible numerical prediction methods. Recent experimental results obtained using a broadband non-intrusive laserbased sound source are presented. The methodology is used to conduct experiments on a wide range of configurations, providing an extensive high-quality validation database. The paper presents an overview of available datasets of dedicated experimental investigations, ranging from simple generic test cases to full 3D aircraft configurations, as well as comparisons with numerical results.
\end{abstract}

Karl-Stéphane Rossignol

German Aerospace Center(DLR), Germany

E-mail: karl-stephane.rossignol@dlr.de

Jan Werner Delfs

German Aerospace Center(DLR), Germany

E-mail: jan.delfs@dlr.de

Michael Mößner

German Aerospace Center(DLR), Germany

E-mail: michael.mößner@dlr.de

Markus Lummer

German Aerospace Center(DLR), Germany

E-mail: markus.lummer@dlr.de

Jianping Yin

German Aerospace Center(DLR), Germany

E-mail: jianping.yin@dlr.de 


\section{Introduction}

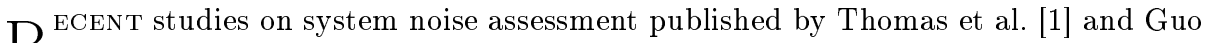
$R$ et al. [2] emphasize the importance of a paradigm change in aircraft design which, combined with modern noise reduction technologies, would allow for a future generation of low-noise airplanes. One important aspect in the above studies is the central role played by noise shielding as a mean of reducing noise radiation towards the ground. One is here particularly concerned with shielding of turbomachinery noise, namely fan and jet noise, as well as installation-related effects, i.e. reflection, diffraction of sound waves and also secondary airframe noise sources. These studies also identified the need for more detailed high-quality experimental databases to provide the necessary background for better system noise analyses.

Early experimental work on the topic were performed in the seventies by Reshotko and collaborators [3-5] at NASA, Hellström [6] in Sweden and Jeffery [7] in England. Reshotko and collaborators [3-5] report on experimental investigations of the "engineover-the-wing" configuration, a possible solution to the conventional, short and reduced take-off and landing (CTOL, STOL and RTOL) noise problem. The selected test configurations consists in various nozzle-wing arrangements of small and large scales with the nozzle installed over the wing to simulate a powered lift configuration or upper surface externally blown flap (EBF) configuration. Flap settings were made to be representative of takeoff and approach settings i.e. $\delta_{F}=30^{\circ}, \delta_{F}=60^{\circ}$ and $\alpha=5^{\circ}$. The effect of nozzle location, jet speed, flow attachment state, existence of internal sources of noise and of the nozzle shape was investigated. The main conclusion of their work is that the noise advantage of the upper wing EBF configuration results primarily from the shielding and redirection, by the wing, of turbomachinery noise. This observation was also found to hold for both conventional engine-over-the-wing configurations and powered-lift configurations. It was found that the wing acts as a good shield for highfrequency noise but generates low frequency noise as the induced flow passes over the trailing edge of the last flap.

Hellström [6], reported experimental results from ground testing of a small fullscale turbofan (with bypass ratio of 2) mounted above shields of various geometry and positions. The shielding characteristics of four different shields, sized to represent wing elements and tailplanes, are investigated. Using special silencers, a separate investigation of intake and exhaust noise was possible. The purpose of this study was to verify the validity of an available prediction method. The authors emphasize the importance of a shield positioning relative to the engine dependent on the source to be attenuated. Intake noise radiates mostly in te upstream direction while exhaust jet noise is mostly important in the rearward radiation arc and is spatially more extended. Low-frequency (below $1 \mathrm{kHz}$ at full-scale) jet noise radiation originates from a region approximately two nozzle diameters downstream of the outlet, while high-frequency $(2 \mathrm{kHz}$ and above at full-scale) jet noise is concentrated approximately half a nozzle diameter downstream of the outlet. Significant attenuation of exhaust noise, on the order of $12 \mathrm{~dB}$ OASPL, and intake noise, on the order of $7.5 \mathrm{~dB}$ OASPL, on the flyover line was achieved.

Jeffery [7] studied the effects of acoustic shielding and flow field refraction both at full-scale in-flight, and at model scale (1/4 full-scale) in the wind tunnel (RAE 24$\mathrm{ft}$ acoustic wind tunnel) The experiments are performed on a Handley-Page 115, a delta-wing research aircraft. In the full-scale experiment the sound source used is a modified Hartman whistle producing a $2.8 \mathrm{kHz}$ tone strong enough to be separated from the engines noise. In the experiment the same Hartman whistle tuned to a fre- 
quency of $10.5 \mathrm{kHz}$ is used. Acoustic measurements are done in the wind tunnel with a microphone traversing horizontally beneath the model at right angles to the flow direction. Measurements are done at different incidence angles to investigate the impact of leading-edge vortices on noise radiation. Their in-flight measurements show a noise attenuation ranging between $10-20 \mathrm{~dB}$ in the shadow region, i.e. beneath the aircraft where the upper side source is not visible. Noise refraction effects are assumed to occur outside the shadow region but could not be acquired in the flight tests, the microphone layout range being too restricted. Previous wind tunnel results indicate a $8 \mathrm{~dB}$ noise reduction $14^{\circ}$ outside the shadow region due to vortex refraction effects [8]. The wind tunnel results give more than $20 \mathrm{~dB}$ attenuation below the aircraft, in the deep shadow region. Sideline noise reductions on the order of $8 \mathrm{~dB}$ are measured. The author relate this reduction to sound wave refraction through interaction with the leading-edge vortex. The transferability of this result to the full-scale flight test data is, however, questioned by the authors and left as an open question.

The first published investigation on acoustic shielding by an unconventional subsonic transport concept aircraft, the blended-wing-body (BWB) configuration, are given by Clark [9]. The authors assessed a $4 \%$ scaled-down model equipped with three engine nacelles in NASA's Anechoic Noise Research Facility at Langley Research center. Extensive noise measurements are done using both traversable and fixed far-field microphones. Flow effects on noise shielding are not considered in this contribution. The reference point source used to simulate broadband engine noise consisted in a four-pipes arrangement supplied with pressurized air [10]. The pipes are bend such that their outlets can be placed close to another, forming four impinging jets. This impinging-jet noise source is broadband between $6 \mathrm{kHz}<f<40 \mathrm{kHz}$. While no attempt is made to replicate engine noise, the source was intended to radiate sound in a frequency range encompassing up to three times the blade passing frequency of a full-scale engine. The source size is such that it can be placed inside the nacelles of the wind tunnel model. Thus, investigations of the effect of the presence of the nacelles over the BWB vs. the BWB alone are made possible with this methodology. The source has a four-lobe pattern azimuthal directivity, with OASPL variations on the order of $2 \mathrm{~dB}$. The four-lobe pattern resulting from the four pipes configuration. Its polar directivity has a preferential lobe pattern away from the pipe assembly where OASPL variation on the order of $3 \mathrm{~dB}$ to $4 \mathrm{~dB}$ are recorded over the range $\theta \pm 45^{\circ}$ relative to the source. An effect obviously related to the presence of the piping in the acoustic field of the source. Their main findings are an overall noise shielding in excess of $20 \mathrm{~dB}$ in the forward sector when all three engines are considered. Aft sector noise attenuation was found to be on the order of $10 \mathrm{~dB}$. An interesting finding is that inlet noise, for the tested configuration, does contribute significantly to the aft noise radiation, through sound wave diffraction. This effect poses a lower limit to the achievable aft noise shielding.

Later, Gerhold [10,11] studied acoustic shielding by a generic wedge-shaped airframe using a similar methodology and without consideration of flow effects. The largest noise attenuation is found, here as well, in the forward sector. The main goal of their work was, however, to validate existing simulation methods for acoustic shielding. An overall good agreement is found between the experiment and the numerical simulations. However, the authors point out the need for better resolved experimental data to be able to deal with more subtle configuration changes. Also, the simulation tools used in the study were found to need refinement, to become more efficient in terms of computational resources, in particular when dealing with high-frequency sound shielding. 
The first European experimental large-scale study dedicated to noise shielding was performed in the framework of the European research project ROSAS, in ONERA's CEPRA 19 anechoic wind tunnel by Ricouard and collaborators [12]. This research was aimed at the assessment of the noise shielding effectiveness of classical airframe components for unconventional aircraft configurations. A complete aircraft model $(1 / 11$ thscale), without nacelle, is used in combinations with a fan and a jet noise simulator to independently assess attenuation by the airframe of both sources. The jet noise simulator is a coaxial exhaust nozzle representative of an advanced high-bypass ratio engine. The fan noise simulator is a turbo-powered simulator (TPS) with a 5.6 inches diameter fan, with 17 blades and 32 vanes, rotated by a 29 blades turbine supplied with gaseous nitrogen. The TPS is installed in a complete nacelle with a symmetric intake. Effects of nozzle location, Mach number variations, and nozzle working conditions (with bypass ratio between 9 and 10) were assessed. Using the jet noise simulator placed over the wing, the authors found a noise attenuation up to $4 \mathrm{~dB}$ at the $90^{\circ}$ emission angle in the flyover plane. When placed over the fuselage, an attenuation on the order of $9 \mathrm{~dB}$ is reached. As expected for jet noise, even higher levels of acoustic shielding are obtained in the co-flow case, up to $6 \mathrm{~dB}$ for the over-the-wing configuration and up to $10 \mathrm{~dB}$ for the over-the-fuselage configuration. The jet noise shielding magnitude increases with an increase in source frequency. Fan noise shielding results gathered with the TPS indicate a strong dependency on source position and working conditions.

Papamoschou and Mayoral [13] conducted generic parametric subscale experiments studies on jet noise shielding on two different configurations. First, using a single-stream Mach 0.9 cold air jet with a rectangular shield. Second, using a dual-stream nozzle with bypass ratio 10 (BPR10) operated at realistic conditions, combined with a hybrid-wing body (HWB) planform shield. Effects of the shield position relative to the nozzles were investigated. Also tabs, chevron and fan flow deflectors were tested in an attempt to compact and redistribute the jet noise source region to reach higher levels of noise attenuation from the HWB planform. In the single-stream nozzle experiment without mixing tabs, an EPNL benefit of $4.4 \mathrm{~dB}$ is achieved. The addition of mixing tabs pushes this benefit up to $6.2 \mathrm{~dB}$ relative to the baseline configuration. The results of this series of measurements on the small-scale single-stream nozzle, compare very well with larger scale experimental data on similar geometries. Investigations done with the BPR10 nozzle showed only marginal jet noise shielding on the order of 1 EPNdB. with the nozzle in its nominal position. A placement 2 nozzle diameter upstream of the nominal position only increased this number to 1.5 EPNdB. An observation which is not surprising considering that the jet noise source extends over 5-6 fan diameters, according to the authors. The above results clearly establish the importance of compaction/redistribution of the jet noise source for an effective noise shielding on the HWB configuration.

Czech and collaborators [14] conducted an extensive large-scale experimental investigation on jet noise shielding by a simplified $4.7 \%$-scale HWB-like configuration. Their aim was to gain an understanding of jet noise shielding effectiveness as a function of engine cycle conditions, location and nozzle configuration. A $4.7 \%$-scale engine simulator with a bypass ratio of 7 , placed over-the-wing, was utilized to perform tests in static and forward flight conditions. Also, the effect of the pylon and its orientation were studied. As noted in [13] in small-scale experiment, placing the nozzle two diameters upstream of the model's trailing edge only provided a limited amount of attenuation, restricted to the high-frequency range. High-frequency jet noise being generated closest to the nozzle outlet plane, whereas low-frequency jet noise occurs up to 5-6 nozzle 
diameters downstream, where the aircraft planform no longer act as a shield. The pylon was found to provide up to $4 \mathrm{EPNdB}$ attenuation in the rear arc while front arc radiation increased by $2-3 \mathrm{EPNdB}$. The use of chevron proved to be an effective mean to modify the jet flow characteristics and shift the source regions closer to the nozzle exit plane. Therefore reducing low-frequency noise by $2-3$ EPNdB inline with results reported in [13]. At the same time chevrons tend to promote high-frequency excess noise which can, however, be effectively shielded by the planform. This study provides the only available large-scale database on jet noise shielding by an HWB-like configuration. A limited amount of testing was also dedicated to shielding of broadband turbomachinery noise. This aspect is assessed using an impinging-pipe device to simulate a broadband point source (see also $[10,11,15]$ for similar applications). Attenuation levels up to $20 \mathrm{~dB}$ are measured in the forward arc.

The most recent experimental study on noise shielding is reported by Hutcheson and collaborators [15]. The study is focused on the investigation of the effect of engine placement and vertical tail configurations on broadband turbomachinery exhaust noise. The experiment was done at a $5.8 \%$-scale HWB configuration in the 14 - by 22 Foot Subsonic Tunnel at NASA Langley Research Center. The model was equipped with broadband engine noise simulators (BENS), consisting of an arrangement of three impinging-jet devices mounted in a streamlined engine nacelle. Only exhaust noise is considered, the BENS intake was capped with a streamlined cover. Noise acquisition was realized using far-field microphones mounted to a traversable support around the whole of the test section. This setup allowed for simultaneous noise measurements on the sideline and on the fly-over line. The authors observed, for the considered BENS noise radiation, that moving the noise simulators further upstream of the TE, using the HWB planform as a shield, does provide an increasing amount of noise attenuation. Up to $13 \mathrm{~dB}$ OASPL (an estimated 3.3 EPNdB) attenuation was measured when the BENS were placed 2.5 nozzle diameters upstream of the TE. The apparent discrepancy between this result and the observations of Papamoschou and Mayoral [13] can be explained through the different compactness of the BENS noise source vs. the elongated region over which jet noise is generated. In the presence of a flow-field, a significant drop in attenuation levels is observed. The authors, postulate that this effect results from sound wave refraction in the near wake region of the HWB model. While vertical tails were found to provide a significant amount of sideline noise shielding, configuration changes only had little impact on the shielding levels.

There exists currently a considerable body of experimental work concerned with the shielding of turbomachinery related noise by aircraft configurations. The trends observed between different sets of experiments is also consistent, even if the methodologies used to do the investigations greatly differs. The determining role played by a particular choice of sound source to perform experiments is emphasized through the range of experimental procedures described above. Each type of source having its own merits. The earliest attempts at investigating noise attenuation by an airframe or airframe model focused mainly on revealing physical trends from in-flight and generic wind tunnel tests. Recent experimental work concentrates on more complex model geometries of the HWB type, motivated by a growing interests in novel unconventional aircraft configurations where noise shielding plays a central role.

Getting back to the system noise analysis of the hybrid wing body aircraft by Thomas et al. [1] and Guo et al. [2]. Because it is currently not possible to provide reliable noise shielding predictions for a fully configured aircraft at realistic flight conditions and frequencies, system noise analyses need to extract the effects of shielding 
directly from available experimental databases. The process is a complicated one, requiring interpolation in the parameter mapping of the experiments to extract data for system noise assessment [2]. The next logical technological step forward is, therefore, to bring numerical simulation methods for shielding effects to a state useful for this kind of analysis. This will be made possible through the availability of high-quality extensive experimental databases for the validation of numerical simulation tools.

With this in mind, a lot of efforts have been put at DLR in the course of the last ten years on the development of a laser-based experimental methodology which can provide very high quality experimental noise shielding data. One central aspect considered in the development of this methodology was the alleviation of the intrusiveness of usual reference noise sources by using a laser-based approach. While most applications of a laser-based reference sound source deal with very high intensity, non-linear, shock-like phenomena, our focus was put on generating an isentropic sound source as a valuable tool to realize dedicated shielding experiments in a non-intrusive manner in wind tunnel of small- to medium-scale. The sound source was found to be well suited for the simulation of engine installation effects, especially because it may be easily used in the presence of flow. Early experimental investigations using the laser-generated sound source were focused on studies using generic bodies. In [16], the shielding characteristics of a cylinder and a thin disc were investigated. The very good agreement of the experimental shielding factors with the analytical solutions for each case provided a high confidence in the experimental methodology. Further, a 3D generic engine nacelle was investigated using the same methodology. The results were compared to simulations based on a boundary element method (BEM), again a good agreement was found. All experiments presented in [16] were done in a quiescent medium. The first experiment on noise scattering done in a uniform flow field and using a laser-based reference sound source were published in [17]. In this experiment, the shielding characteristics of a generic NACA 0012 two-dimensional wing are determined, in quiescent air as well as under flow conditions, in an open-jet wind tunnel environment. In a later experiment, the results presented in [18] show the scattering characteristics of generic spheres of various sizes and materials, both in quiescent air and uniform flow. A comparison is made between the experimental results and the analytical solution showing an excellent agreement. The most recent investigations done with the laser-based sound source methodology are concerned with the investigation of realistic 3D unconventional aircraft configurations [19].

The remaining of the article will provide some details on the physical principles of the laser-based sound source as well as an overview of available databases acquired using the technique. The discussion presented in section 2.1 is concerned with the laserbased impulsive sound source. It was presented at the 22nd AIAA/CEAS Aeroacoustics Conference 2016 (manuscript AIAA-2016-2795 [17]). An extensive discussion of the source properties can be found in $[20,17,19]$. The discussion presented in section 3 is concerned with basic experiments on generic bodies aiming at providing a confirmation of the validity of the experimental methodology. A review of some results taken from $[16,18]$, is presented. The results from [16] were presented at the 15th AIAA/CEAS Aeroacoustics Conference in 2009 (manuscript AIAA-2009-3329-624). Results taken from [18] were presented at the 42nd European Rotorcraft Forum in 2016 (manuscript nr. 127). In section 4 , new results are presented extending earlier work from $[17,19]$. 


\section{Experimental and numerical approaches}

\subsection{Laser-Based Impulsive Sound Source}

By focusing a high energy laser beam to a point i.e. an effective air volume, it is possible to initiate the formation of a small plasma which rapidly expands [21,22], thus forming a pressure wave about its boundary which propagates through the surrounding medium. From the wave equation for the pressure perturbation $p^{\prime}$ in a stagnant medium of variable mean density $\rho^{0}(x)$ the above phenomenon can be described by [20],

$$
p^{\prime}(\boldsymbol{x}, t)=\frac{(\gamma-1)\left(1-M^{2}\right)}{4 \pi a_{\infty}^{2}\left(\sqrt{\left(\boldsymbol{M} \cdot \boldsymbol{r}_{0}\right)^{2}+\left(1-M^{2}\right) \boldsymbol{r}_{0}^{2}}-\boldsymbol{M} \cdot \boldsymbol{r}_{0}\right)} \frac{\partial \vartheta_{p}}{\partial \tau}=\frac{(\gamma-1)}{4 \pi a_{\infty}^{2} r_{0}^{+}} \frac{\partial \vartheta_{p}}{\partial \tau}
$$

where all variables are to be evaluated at the retarded time $\tau$, which is related to the observer time $t$ by

$$
t=\tau+\frac{1}{a_{\infty}\left(1-M^{2}\right)}\left\{-\boldsymbol{r}_{0} \cdot \boldsymbol{M}+\sqrt{\left(\boldsymbol{r}_{0} \cdot \boldsymbol{M}\right)^{2}+\left(1-M^{2}\right) r_{0}^{2}}\right\}
$$

In Eqs. (1) and (2), the distance $r_{0}(\tau)$ is the magnitude of the vector from source $\boldsymbol{\xi}_{0}(\tau)$ to observer $\boldsymbol{r}_{0}:=\boldsymbol{x}-\boldsymbol{\xi}_{0}$ at emission time $\tau$. Equation 1 emphasizes the importance of the temporal heat input $\left(\frac{\delta \vartheta_{p}}{\delta \tau}\right)$ in generating a high amplitude pressure wave. Moreover such a pressure wave does not exhibit any directionality and decreases linearly away from its origin.

To achieve plasma formation, a minimum amount of energy as to be provided into the medium; the energy threshold $\left(E_{t h r}\right)$ for the initiation of plasma formation. Once $E_{t h r}$ is reached, the plasma starts building up and its temperature and density increases greatly while absorbing a large portion of the input laser beam energy [23, 24]. A theoretical description of the phenomenon is provided by the multiphoton ionization and cascaded ionization mechanisms [25]. In its early stage, the expanding plasma generates a pear-shaped pressure front with initial supersonic propagation speed which becomes an almost omni-directional pressure wave in the far-field [25,26]. The initial shock wave slows down to the isentropic speed of sound after approximately $20 \mu \mathrm{s}$ at which point it propagates as an isentropic acoustic wave. Consequently the small plasma generated can be seen as a breathing sphere with $10 \mathrm{~mm}$ radius [27]. The value of $E_{t h r}$ is of about $3.5 \times 10^{12} \mathrm{~W} / \mathrm{cm}^{2}$ for an irradiation of wavelength $\lambda=532 \mathrm{~nm}$ in air and at standard atmospheric pressure of $101.3 \mathrm{kPa}$ [28]. Threshold energy is dependent on the type of gas, its pressure, the radiation wavelength, the duration of the laser pulse and its focusing conditions [25]. Most relevant for the current practical application, are the last two parameters.

The shape and volume of the plasma spot are strongly dependent on the angle with which the laser beam is focused. For a given input beam diameter, the longer the focal length, the longer the plasma spot will be along the beam axis. The same is also true for the minimum beam diameter achievable at the focal point. A formal description of these observations is given by the theory of Gaussian beams [29]. Even if the output beam profile of a Nd:YAG laser is not perfectly Gaussian, due to its multi-mode structure, a Gaussian beam approximation is still a good representation.

The parameters of a Gaussian beam in the waist region are given in figure 1 (see also [29]). For an ideal Gaussian beam, the beam divergence is given by 


$$
\theta_{0}=\frac{2}{\pi} \frac{\lambda}{2 W_{0}},
$$

with $W_{0}$ the minimum beam radius. For a convex lens placed at the collimated beam waist, the resulting focussed beam waist is,

$$
W_{0}^{\prime} \approx \frac{\lambda}{\pi W_{0}} f_{L}=\theta_{0} f_{L} \text { and } 2 W_{0}^{\prime} \approx \frac{4 \lambda}{\pi} F_{\#} \approx \frac{4 \lambda}{\pi} \frac{f_{L}}{D},
$$

where $D=2 W_{0}$ and $f_{L}$ the lens focal length. The depth of focus is given by,

$$
2 z_{0}^{\prime}=\frac{2 \pi W_{0}^{2}}{\lambda}
$$

with $z_{0}$ the Rayleigh length. From equation 4, to ensure the highest possible energy densities, one needs to minimize $2 W_{0}^{\prime}$ by minimizing the ratio $f_{L} / D$. This can be achieved through a shorter $f_{L}$, by expanding the input beam, $D$, or a combination of both to effectively increase the focusing angle of the input beam.

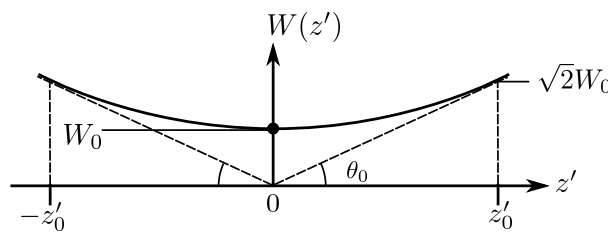

Fig. 1: Gaussian beam parameters in the waist region. $W\left(z^{\prime}\right)$ is the beam radius vs. position $z^{\prime}$ along the beam axis. The minimum beam radius at $z^{\prime}=0$ is $W_{0} . W\left(z^{\prime}\right)$ increases linearly for large values of $z^{\prime}[29]$.

Laser-based sound generation enables the production of a nearly omni-directional (see figure 2) broadband pressure wave in a non-intrusive manner [27]. In figure 2, the source sound pressure level $\left(L_{p}\right)$ reduced by the average sound pressure level $\left(\overline{L_{p}}\right)$ over the complete emission angle range is plotted vs. emission angle and for both the $y-z$ and $\mathrm{x}-\mathrm{y}$ planes. The generated pressure wave is broadband over the useful frequency range $1 \mathrm{kHz}<f<80 \mathrm{kHz}$, with a spectral maximum at approximately $30 \mathrm{kHz}$.

\subsection{Numerical approach for 2D shielding calculations}

The 2D acoustic simulations are performed with the DLR PIANO Code [30]. This code solves the acoustic perturbation equations on curvilinear block-structured meshes with a 4th order DRP (dispersion relation preserving) scheme for space discretization. The background mean flow needed by the solver is calculated with the DLR TAU-Code [31] which solves the RANS-equations with a $\mathrm{k}-\omega$ turbulence model as closure. The far field conditions are adapted to the experimental setup (Mach number: 0.16, Reynolds number based on chord length: $\left.0.8 \times 10^{6}\right)$.

The blocks of the computational mesh for the acoustic solution are shown in figure 3 . The mesh contains about $5 \times 10^{6}$ grid points and can resolve frequencies up to $80 \mathrm{kHz}$. The outer boundaries are defined as radiation boundaries, the airfoil surface is defined as an adiabatic slip wall. 


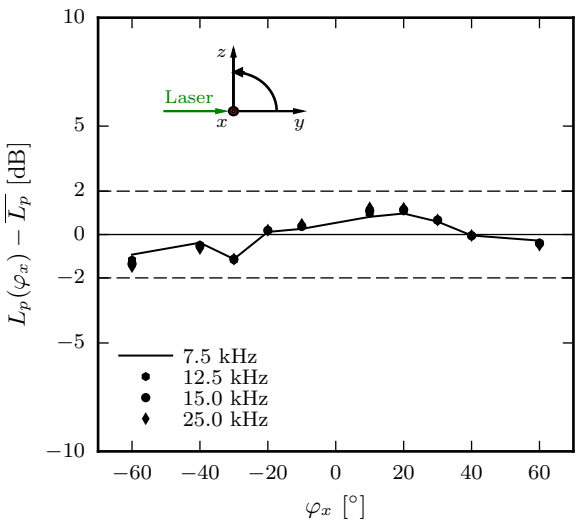

(a) y-z plane, rotation about $\mathrm{x}$-axis $\left(\varphi_{x}\right)$

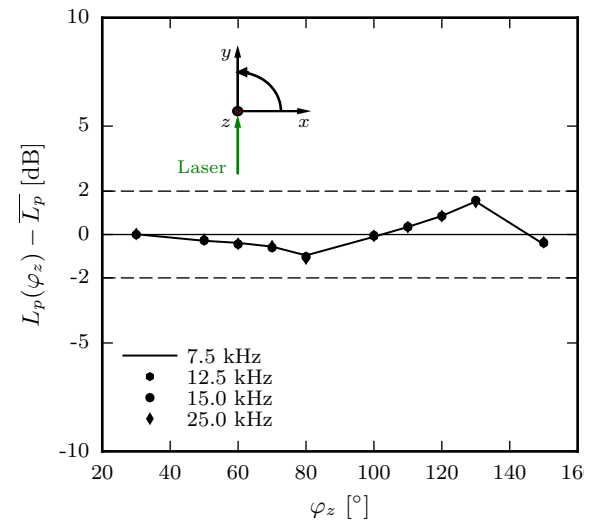

(b) x-y plane, rotation about z-axis $\left(\varphi_{z}\right)$

Fig. 2: Laser sound source directivity vs. frequency

The sound source utilized in the computations is defined as a point heat source. It locally adds heat into the computational domain. The point source is set up to follow the mean flow and it exists only over a short period of time. Its heat input profile vs. time $\left(\vartheta_{p}(\tau)\right)$ is derived from measurements of the isolated source (see Fig. 5b) making use of Eq. 1. Data reduction is done in agreement with the experimental procedure.
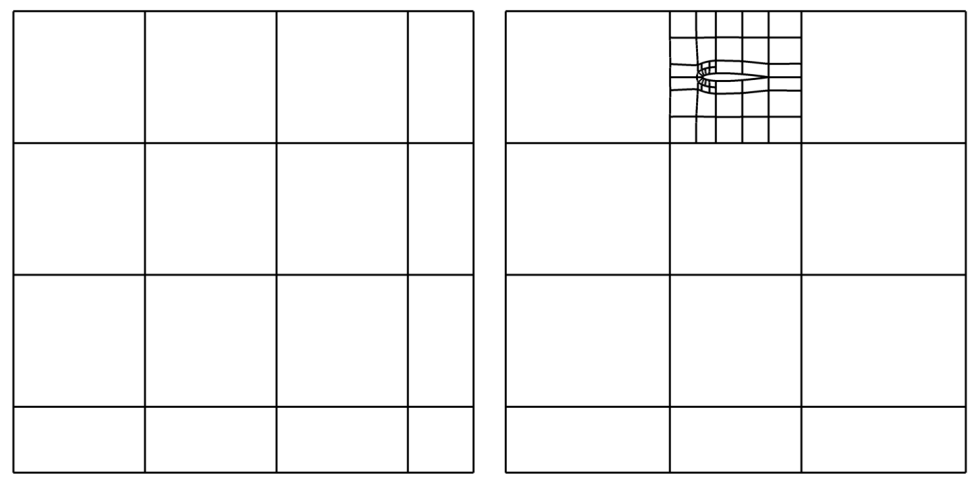

Fig. 3: Blocks of the computational meshes for obtaining the shielding results of the NACA 0012 2D wing. Left hand side: Reference case without geometry, right hand side: case with NACA 0012 airfoil

The typical time trace (without microphone correction applied) of the laser-generated pressure waves, one meter away from the sound source, are shown in Figure 4a. In figure $4 \mathrm{~b}$, the corresponding spectra are plotted. The source displays an excellent repeatability of the generated pressure pulses (figure 4a). In figure 4a, a total count of 252 pulses is plotted along with a histogram of the maximum pulse amplitude i.e. num- 
ber of pulses vs. amplitude in Pascal. Similarly, in figure 4b, 252 spectra are shown along with the histogram of their respective overall sound pressure levels (OASPL). Once microphone corrections are applied, the actual pressure pulse of the source can be determined (shown in figure 5a) [17] and the related source function $\vartheta(\tau)$ can be calculated, i.e. power vs. time, applied by the laser to the medium for the generation of a pressure pulse (figure $5 \mathrm{~b}$ ). The curve of $\vartheta(\tau)$ in figure $5 \mathrm{~b}$, can be easily approximated through a Gaussian function and implemented in numerical simulation codes.

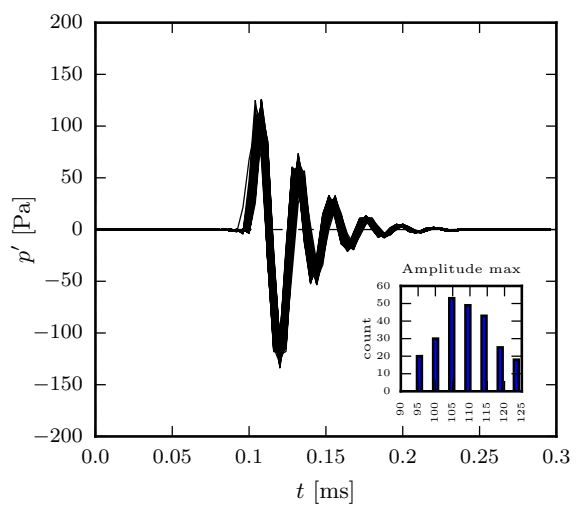

(a) Individual time traces

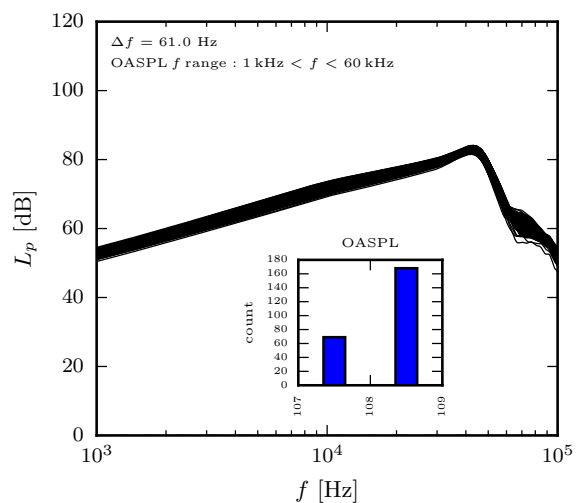

(b) Individual spectra

Fig. 4: Repeatability of the laser generated pressure waves.

Because the source pressure pulse is of very short duration $(\approx 0.1 \mathrm{~ms})$, very high sampling rates are needed for an adequate acquisition of the pressure signals. The data presented in the next sections were, therefore, acquired using GMB Viper systems operated at their maximum sampling rate of $250 \mathrm{kHz}$ with an anti-aliasing filter cutoff frequency fixed at $100 \mathrm{kHz}$. This setup enables a correct sampling of signals with frequencies up to approximately $100 \mathrm{kHz}$. To cope with the short period of the pressure pulse, 1/8" G.R.A.S. 40 DP pressure field microphones with a $140 \mathrm{kHz}$ dynamic range were used. In all experiments, the laser is operated at full power and at a fixed repetition rate of $10 \mathrm{~Hz}$. If not otherwise mentioned, measurements are performed in-flow, using a Bruel \& Kjær nose cap.

The experiments are performed with a continuously running laser operated at full power, in order to get the maximal possible signal to noise ratio (SNR). The laser flash lamp trigger signal is simultaneously recorded to facilitate data post-processing. In cases where $U_{0}>0 \mathrm{~m} / \mathrm{s}$, the time signals have to be band-filtered between $3 \mathrm{kHz}$ to $6 \mathrm{kHz}$ and $110 \mathrm{kHz}$, at $M_{0}=0.087$ and $M_{0}=0.16$ respectively, to remove lowfrequency contamination of the data through flow-induced noise on the microphone body as well as from vibrations of the microphone support. Filtering of the raw time series is necessary for a better extraction of the pulses during post-processing, especially when investigating the shielded sound field, where the absolute amplitude of the sound pulses are greatly reduced. Fourier analysis, of the individual and averaged pulses, is performed using non-overlapping blocks zero-padded to a total length of 4096 samples 


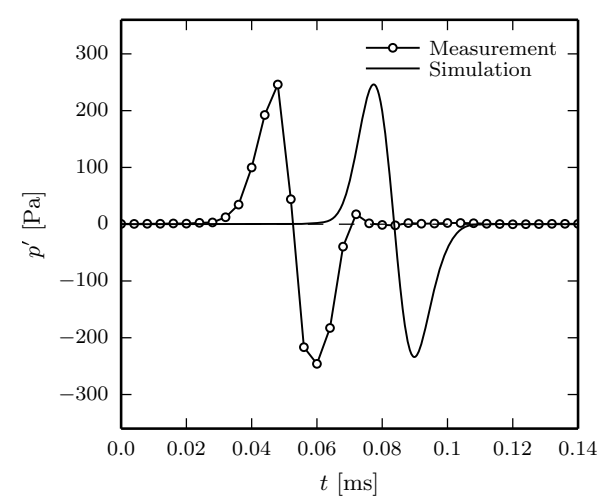

(a) Averaged pulse shape. Experiment vs. simulation.

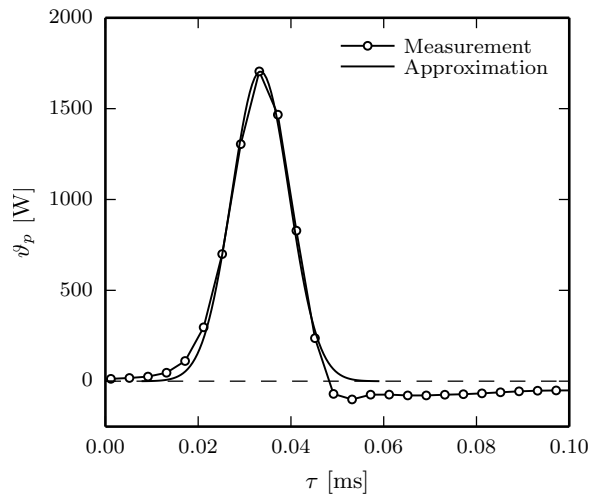

(b) Source function. Experiment vs. approximation.

Fig. 5: Left, averaged experimental pulse shape at $R=0.2 \mathrm{~m}$ vs. simulated pulse. The simulated pulse is shifted on purpose for clarity's sake. Right, experimental source function (o) and its corresponding approximation through a Gaussian function. The simulated pulse in (a), is computed using the CAA code PIANO [32] with the approximated source function in (b) as input.

for a frequency resolution of $\Delta f=61 \mathrm{~Hz}$. This block length is kept constant throughout the post-processing to ensure the complete recovery the signals when measuring with the shielding object installed. Also, a constant and consistent block length has to be defined for a correct representation of the pulses energy content in both the shielded and free-field cases. A necessity, for the obtention of correct shielding factors. Finally, no window function is applied to the signals prior to the FFT computations as the pulses are short-time signals which tend to zero quickly towards each block bounds.

\section{Assessment of Noise Shielding by Generic Bodies}

In this section results of investigations done at simple generic bodies are presented. The purpose of these simpler tests is to establish the validity and capacity of the experimental methodology. In the discussion below, the narrow band spectrum of the shielding factor is defined as

$$
\eta(f)=\frac{\left|\hat{p}_{s}\right|}{\left|\hat{p}_{i}\right|}
$$

with $\left|\hat{p}_{s}\right|$, the modulus of the Fourier-transformed shielded acoustic signal and $\left|\hat{p}_{i}\right|$, the modulus of the Fourier-transformed incident acoustic signal. The narrow-band shielding level is given by

$$
\gamma_{p}(f)=20 \log (\eta)
$$


The incident and shielded acoustic signals used to compute the shielding factor, $\eta$, at a location of interest, account for sound wave attenuation due to geometrical spreading.

Some of the database presented in this section were acquired in a non-anechoic (hard-wall) room. The data acquired in this environment was, however, confirmed to still obey a $1 / R^{2}$ decay law, as in an anechoic environment. The data presented in Fig. 6 for the anechoic room case were collected in DLR's Acoustic Wind Tunnel Braunschweig (AWB). The AWB is an open-jet facility with a cross section of $1.2 \mathrm{~m} \times 0.8 \mathrm{~m}$ and capable of running at speeds of up to $65 \mathrm{~m} / \mathrm{s}$. It is optimized for acoustic measurements at frequencies above $250 \mathrm{~Hz}$. A comparison between data acquired in both environments is presented in figure 6 . In that figure, the summed SPL $\left(L_{\text {sum }}\right)$ in the frequency range $5 \mathrm{kHz} \leq f \leq 25 \mathrm{kHz}$, normalized by the summed level at $R=0.325 \mathrm{~m}$, is plotted against distance from the source center. Because of the short duration of the source acoustic pulse, it is possible to separate the incident wave from its reflection on surrounding objects or on the walls. Therefore, the sound field in the measurement range is effectively unaware of the surrounding environment.

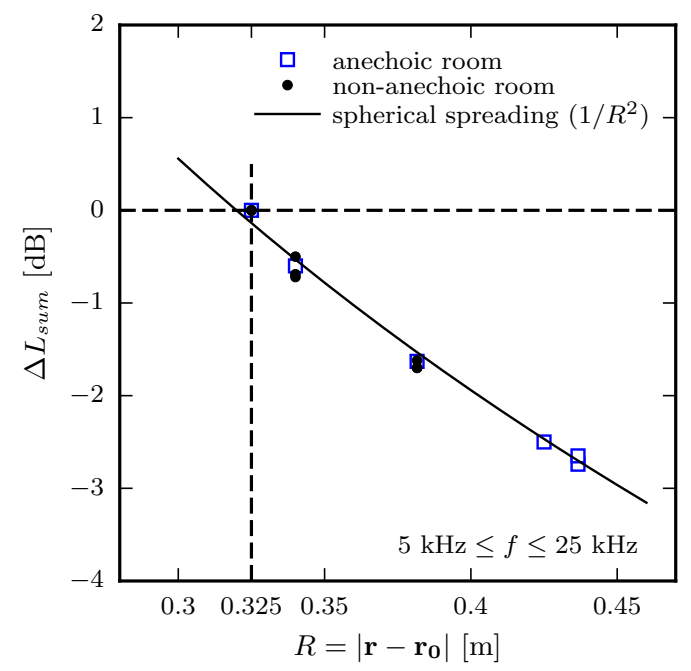

Fig. 6: Relative decay of the source summed SPL, in the range $5 \mathrm{kHz} \leq f \leq 25 \mathrm{kHz}$, with distance from the its center, $R=\left|\mathbf{r}-\mathbf{r}_{\mathbf{0}}\right|$. Anechoic vs non-anechoic environments. $\mathbf{r}$ is the microphone position vector and $\mathbf{r}_{\mathbf{0}}$ is the source position vector.

The DLR sound shielding prediction tool used in the generic test cases (circular disc, long cylinder, and isolated nacelle) was the boundary element code BEMPAR [16]. It solves the exterior Helmholtz problem (scattering problem) based on the BurtonMiller approach, cf. [35,34]. The surface of the scattering geometry is triangulated and a constant pressure value is assumed on each plane triangle. One needs at least 6 elements per wavelength and the minimum wavelength that can be calculated for a body with surface $A$ discretized by $N$ elements is $\lambda_{\min } \approx 6 \sqrt{A / N}$. The surface triangulation was generated using the netgen code [38]. The FORTRAN subroutines of [34] for the calculation of the discretized integral operators were reimplemented in 
$\mathrm{C}++$ and the resulting system of linear equations is solved using parallel linear algebra routines from the ScaLAPACK software package ${ }^{1}$. Details of the algorithm can be found in [16]. Since the full matrix must be stored BEMPAR is limited to small frequencies for large geometries and has been superseded at DLR by the Fast-Multipole code FMCAS.

\subsection{Test Case 1: Disc [16]}

The first experimental test case is shown in figure $7 \mathrm{a}$. It consists of a basic circular flat plate with a diameter of $40.2 \mathrm{~cm}$ and $3 \mathrm{~mm}$ thickness. The plate is held from the bottom by a metal rod of $1.2 \mathrm{~cm}$ in diameter and $80 \mathrm{~cm}$ long. Microphones are placed at a distance of $25 \mathrm{~cm}$ relative to the center of the plate and are $10 \mathrm{~cm}$ apart, the first one being in front of the plate center (see figure $7 \mathrm{~b}$ ). The laser beam comes perpendicularly to the microphones axes and its focus point is aligned with the plate center, $7.5 \mathrm{~cm}$ away from it $(32.5 \mathrm{~cm}$ from the first microphone tip).

A comparison of the computed (BEM) and measured shielding factors in the range 5 to $25 \mathrm{kHz}$ for the circular plate case is shown in figure 8 . A very good agreement is found for microphone \#2 with variations on the order of $1 \mathrm{~dB}$ except where attenuation peaks exists. Overall the shape of the shielding factor spectrum is well captured in the considered range. For microphone \#1, directly behind the plate center, we observe a very different spectrum with a peak value of approximately 0.85 at $5 \mathrm{kHz}$ and less fluctuations of the shielding factor. These observations matches well with the theory of wave diffraction by a circular plate which also predict a concentrated region of high pressure directly behind the circular plate center point, called Arago spot.

For this microphone position, the agreement between experiment and computation is found to be not as good as for microphone \#2. It is hypothesized that position uncertainties alone are responsible for these differences. In fact, a second computed shielding factor spectrum for a slightly eccentric position $(r=0.005 \mathrm{~m})$ is also plotted in figure 8 showing a much better agreement between experiment and computation $(\approx 1$ $\mathrm{dB})$. This reveals the strong spatial dependency of the shielding factor in the central region behind the plate which was furthermore confirmed from the BEM shielding factor maps (see figure $8 \mathrm{~b}$ ).

The surface of the circular flat plate was discretized with up to 51500 triangles, in order to allow calculations up to $25 \mathrm{kHz}$. The memory requirements were about $45 \mathrm{~GB}$ for single precision calculations.

\subsection{Test Case 2: High Aspect-Ratio Cylinder [16]}

The cylinder setup is shown in figure 9. As for the circular plate, it is held from the bottom, through its axis, by a metal rod of $2 \mathrm{~cm}$ diameter and $59.5 \mathrm{~cm}$ long. The cylinder itself has a diameter of $8 \mathrm{~cm}$ and is $80 \mathrm{~cm}$ long. For this test case, the same microphone support as before is used but with a different microphone alignment. The second microphone is aligned with the geometrical center of the cylinder and both microphones 1 and 3 are $10 \mathrm{~cm}$ apart from the central one (see figure $9 \mathrm{a}$ ). The laser spark is generated $11.5 \mathrm{~cm}$ away from the cylinder geometrical center and is $40.5 \mathrm{~cm}$ away from the second microphone.

\footnotetext{
1 http://www.netlib.org
} 


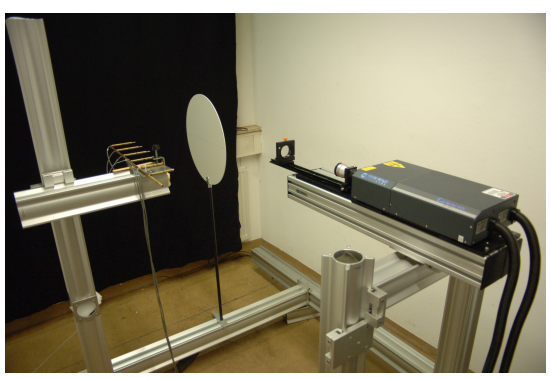

(a) Experimental setup.

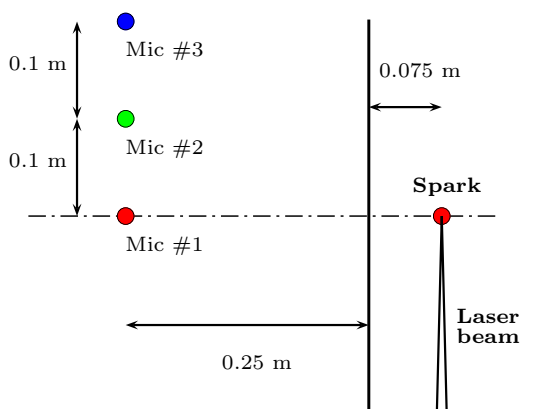

(b) Schematic disposition of all elements as seen from the top. (Not to scale)

Fig. 7: Experimental setup, circular plate test case

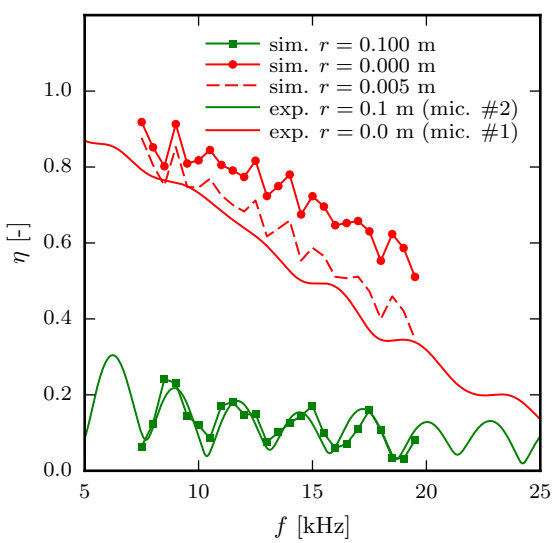

(a) Spectrum of $\eta$, experiment vs. simulations. Experimental data for microphone \#1 and \#2 of Fig. 7a.

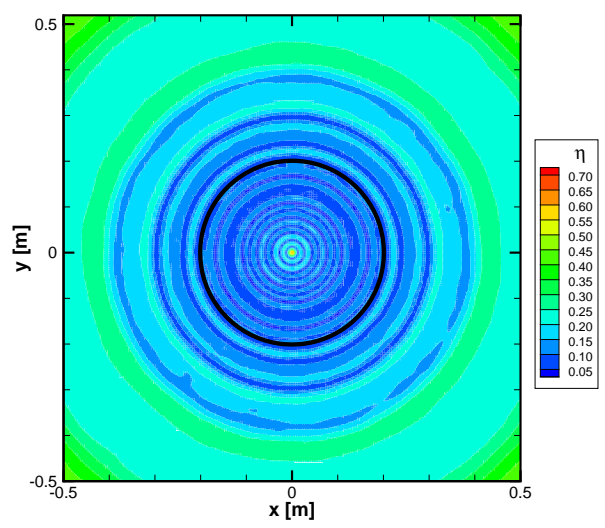

(b) Computed shielding factor in the plane of the microphones

Fig. 8: Shielding factor, comparison between experiment and simulations

For the cylinder test case, a comparison of the shielding factor for microphone \#1 and \#2 in the range 5 to $25 \mathrm{kHz}$ is plotted in figure 10 . Microphone \#3 is ignored because of the setup symmetry. In the present case the measured shielding factor at microphone \#1 is also seen to agree very well with the BEM computation with overall variations of less than $1 \mathrm{~dB}$. Here remember that microphone \#1 is located $10 \mathrm{~cm}$ $(1.25 D)$ away from the central axis. The comparison at microphone \#2 is not as good but still presents maximum differences of the order of $1 \mathrm{~dB}$ which is comparable to the results presented in figure 8 for the circular plate case. Here it is once again important to emphasize that positioning of the microphone is a critical aspect. A zone 


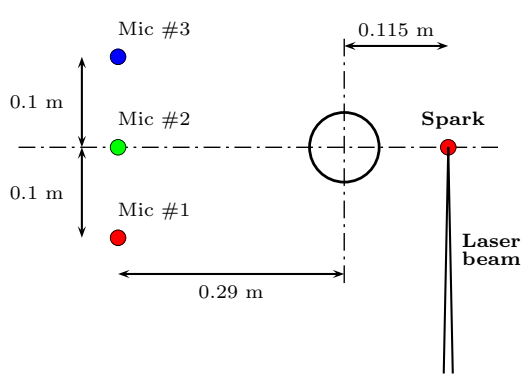

(a) Schematic disposition of all elements as seen from the top. (Not to scale)

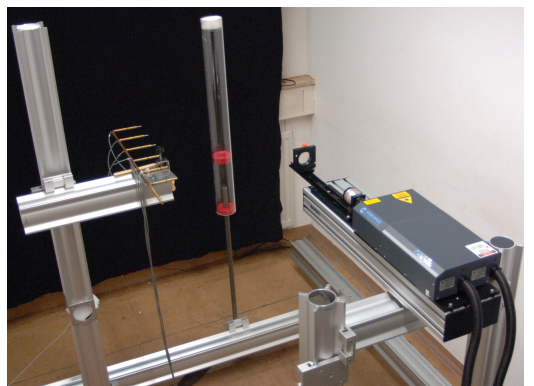

(b) Experimental setup for the cylinder test case.

Fig. 9: Long cylinder test case.

of large shielding factor gradients exists behind the cylinder as shown from the BEM computations 2D maps (see figure 10b).

The surface of the high aspect-ratio cylinder was discretized with up to 56500 triangles, in order to allow calculations up to $25 \mathrm{kHz}$. The memory requirements were about 55 GB for single precision calculations.

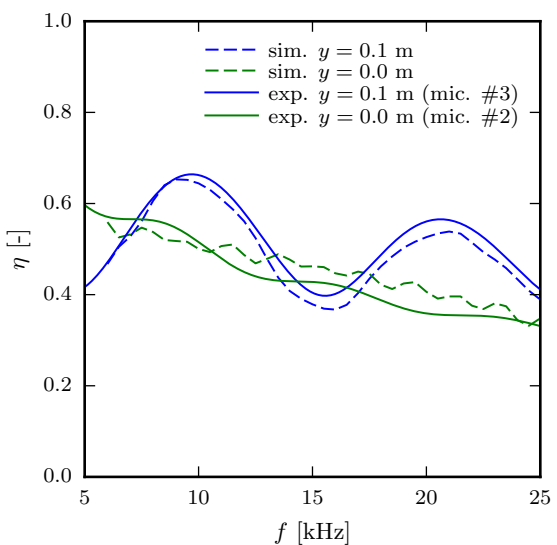

(a) Spectrum of $\eta$, experiment vs. simulations. Experimental data for microphone \#2 and \#3 of Fig. 9a.

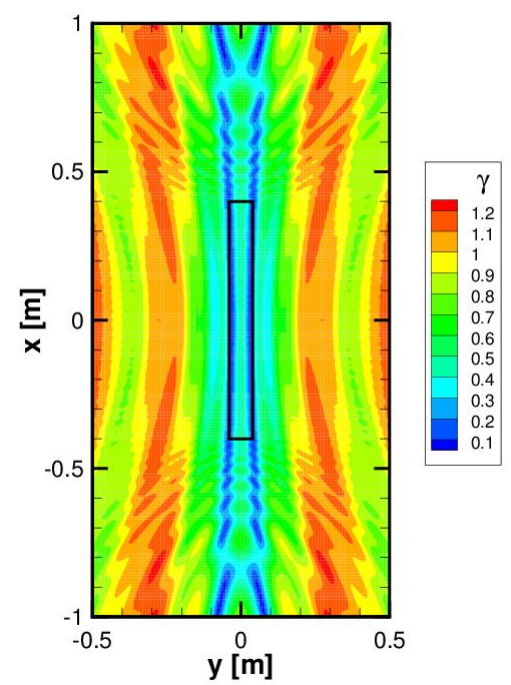

(b) Computed shielding factor in the plane of the microphones. Cylinder geometry shown by thick black rectangle.

Fig. 10: Shielding factor, comparison between experiment and simulations 


\subsection{Test Case 3: Spheres [18]}

This test case was considered in the GARTEUR Action Group HC/AG-24 dealing with acoustic scattering of spheres. The Action Group was established to address noise scattering by helicopter rotors in the presence of the fuselage. This test case is a necessary first step to help establishing an appropriate test setup for acoustic scattering of a generic GARTEUR helicopter model. The experiments were conducted in DLR's AWB tunnel in Braunschweig.

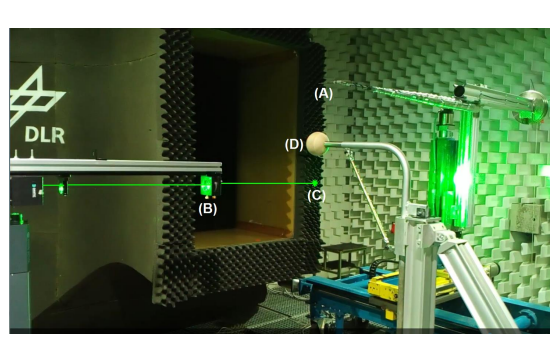

(a) Test setup overview

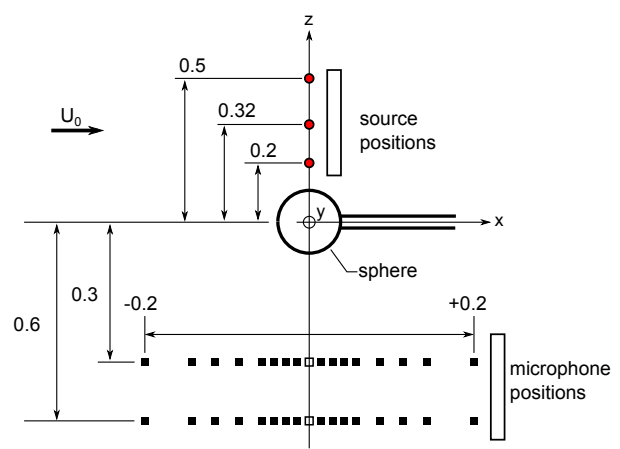

(b) Measurement positions

Fig. 11: Test setup in AWB. (A) microphone, (B) laser optics, (C) plasma spot, (noise source), (D) sphere

Two sphere sizes, a small one with $\mathrm{D} 1=0.12 \mathrm{~m}$ diameter and a big one with $\mathrm{D} 2=$ $0.34 \mathrm{~m}$ diameter are used. The size of the spheres are derived according to the largest dimension of the BO105 fuselage with 1/12.5 scale in both lateral- and streamwise directions. Here the scale factor of 12.5 is chosen to insure that the scaled rotor noise frequencies fit inside the effective frequency band of the noise sources. For the smaller sized sphere D1, a wooden (beech) and an aluminum sphere were tested to determine the influence of the different material impedance. Measurements are done at three wind speeds of $0 \mathrm{~m} / \mathrm{s}, 30 \mathrm{~m} / \mathrm{s}$ and $45 \mathrm{~m} / \mathrm{s}$ corresponding to Mach numbers $M=0,0.09,0.13$ respectively. Comparisons of the test results with the analytical solution for sound scattering by spheres is used to verify the accuracy of the experimental results

An excerpt of the results is given in figure 12 for one sphere diameter $(D=0.12 \mathrm{~m})$ and two frequencies $(f=7.5 \mathrm{kHz}$ and $15 \mathrm{kHz}$ ). In figure 13 , a comparison is made between BEM simulations and experiment at $M=0.13$ for the same test case. The experimental results are found to be in good overall agreement with the analytical solution and the BEM simulation. Some more important discrepancies at $f=15 \mathrm{kHz}$ can be seen between the measurements and the analytical solution. These are attributed to the sting used in the experiment, which was not accounted for in the computations.

The laser-based non-intrusive sound source utilized in this study allows a direct measurement of the shielding factors both in quiescent and moving mediums. The noise shielding results provide clear and consistent trends for all cases considered. The dependency of the shielding factor on frequency or sizes of the sphere or support system 


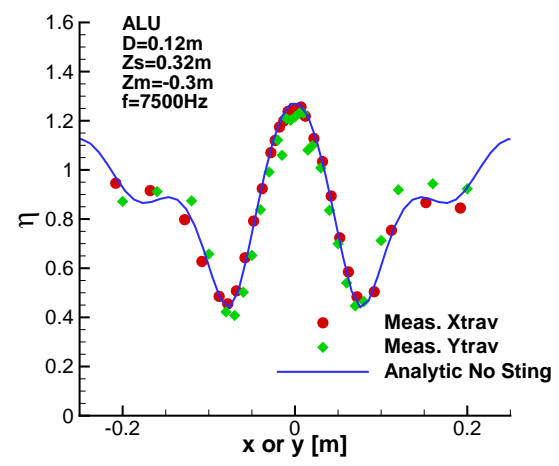

(a) $f=7.5 \mathrm{kHz}$

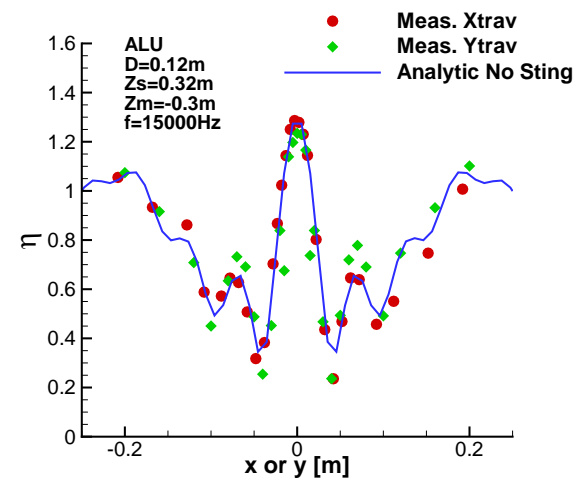

(b) $f=15.0 \mathrm{kHz}$

Fig. 12: Shielding factor, comparison between experiment and analytical solution. Aluminum sphere, $\mathrm{D}=0.12 \mathrm{~m}, M=0$.

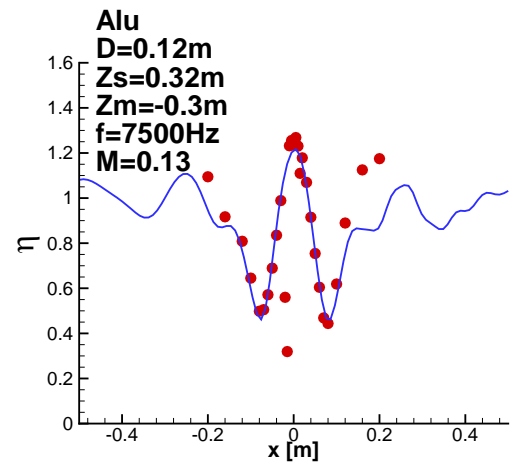

(a) $f=7.5 \mathrm{kHz}$

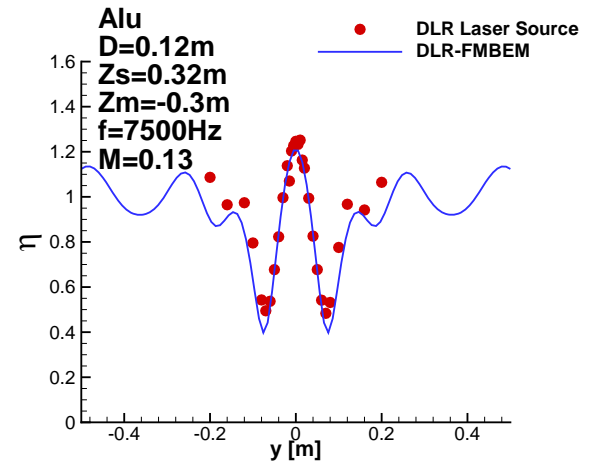

(b) $f=15.0 \mathrm{kHz}$

Fig. 13: Shielding factor, comparison between BEM simulations and experiment. Aluminum sphere, $\mathrm{D}=0.12 \mathrm{~m}, M=0.13$.

are captured. The influence of the sting on the shielding factors of the sphere cannot be ignored for the small sphere. Therefore when using the test data for the purpose of the code validation, the sting influence needs to be considered in the numerical simulation.

\subsection{Test Case 4: Isolated Nacelle [16]}

The experimental setup used for this test case is shown in figure 15. A simple generic streamlined engine nacelle is used to evaluate the ability of the experimental method- 
ology for the investigation of a more complex diffracted sound field. The laser is focused inside the nacelle, at its center, to generate an outgoing sound wave which gets diffracted at the nacelle inlet and outlet. The measured shielding factor spectrum (in the range $5-25 \mathrm{kHz}$ ) along with the corresponding BEM computations are presented in figure 16 for angles $\theta=12^{\circ}, 17^{\circ}, 22^{\circ}, 32^{\circ}, 42^{\circ}, 52^{\circ}$.

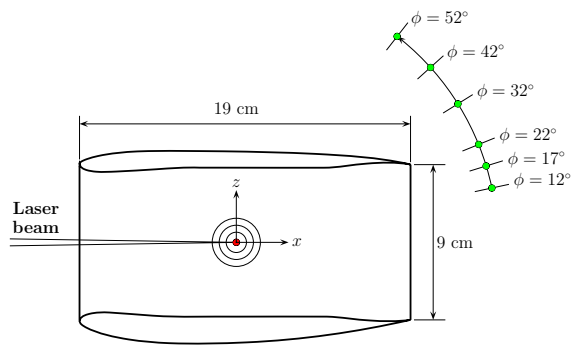

(a) Top view

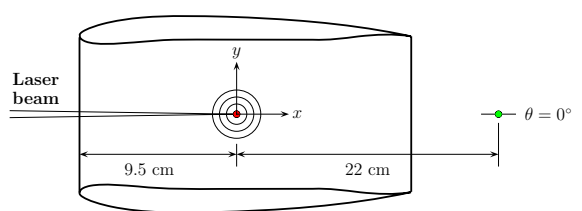

(b) Side view

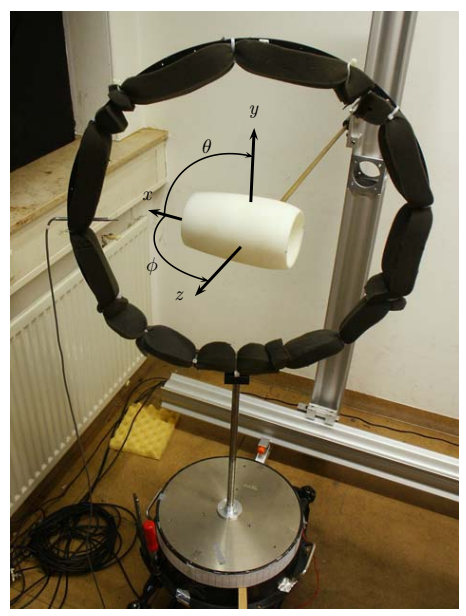

Fig. 15: Experimental setup for the generic nacelle test case. The laser beam is coming from the right-hand side of the picture.

A good overall agreement of the numerical and experimental spectra is observed in the range $22^{\circ}<\theta<52^{\circ}$. The overall spectral shape of both measurements and computations match well over the whole frequency range. The absolute peak levels (spikes) of $\eta$ do not, most of the time, compare well with the simulations. The spatial resolution limit of the microphone is probably reached, thus rendering lower levels. Between the peaks, levels trends are correctly recovered. At $\phi=12^{\circ}$ and $17^{\circ}$, the quality of the agreement between measurements and computation is not good. For these three measurement angles, one can clearly observe that the similarity between both spectra worsen as frequency increases. The spatial distribution of the shielding factor at those angles is characterized by very sharp gradients (simulation results, shown in figure 17) which cannot be resolved in the experiment. The comparison between simulation and experiment is thus, when looking at single frequency band, very sensitive to slight position differences between experiment and simulation.

The surface of the isolated nacelle was discretized with up to 46000 triangles, in order to allow calculations up to $25 \mathrm{kHz}$. The memory requirements were about $37 \mathrm{~GB}$ for single precision calculations. 


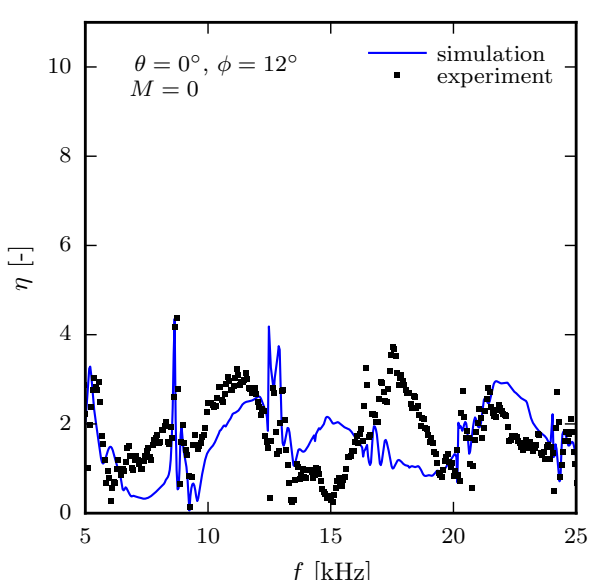

(a) $\theta=0^{\circ}, \phi=12^{\circ}$

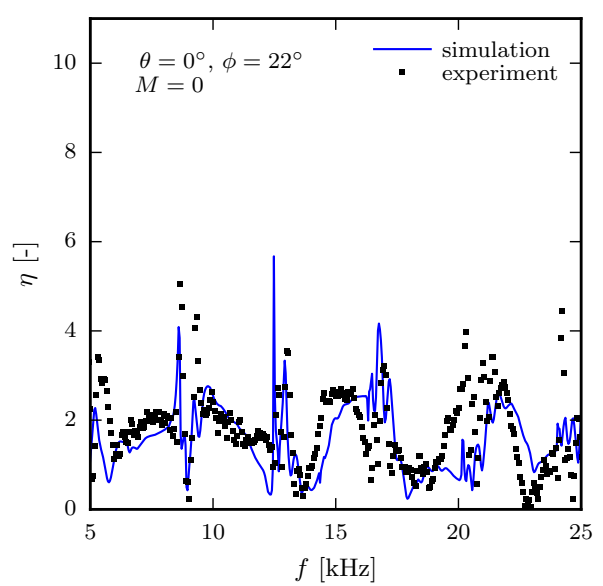

(c) $\theta=0^{\circ}, \phi=22^{\circ}$

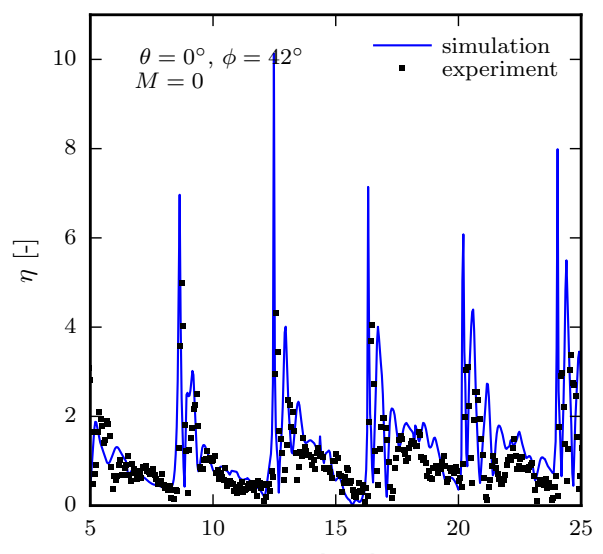

(e) $\theta=0^{\circ}, \phi=42^{\circ}$

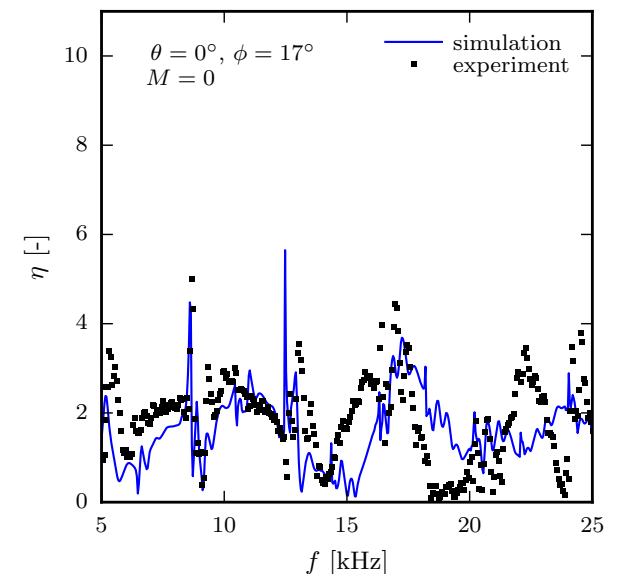

(b) $\theta=0^{\circ}, \phi=17^{\circ}$

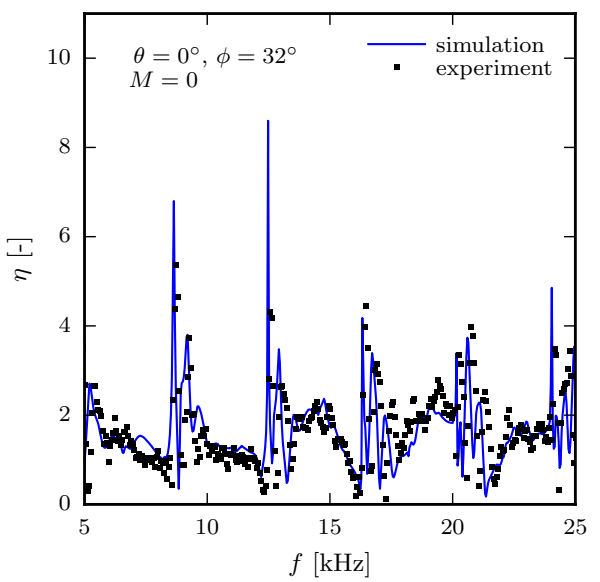

(d) $\theta=0^{\circ}, \phi=32^{\circ}$

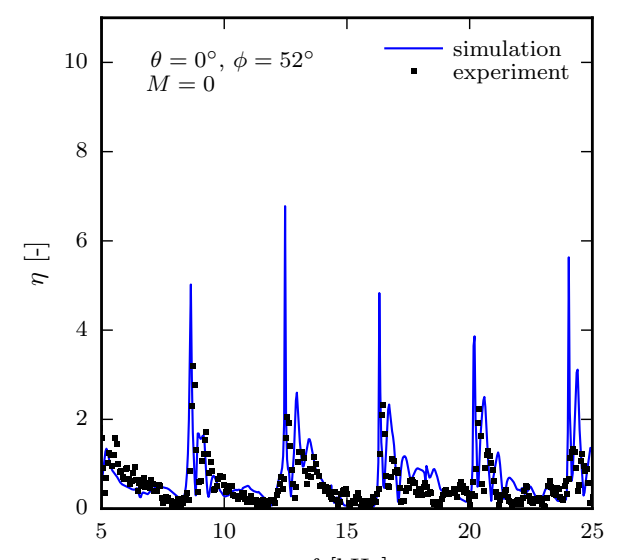

(f) $\theta=0^{\circ}, \phi=52^{\circ}$

Fig. 16: Shielding factor, comparison between experiment and simulations 


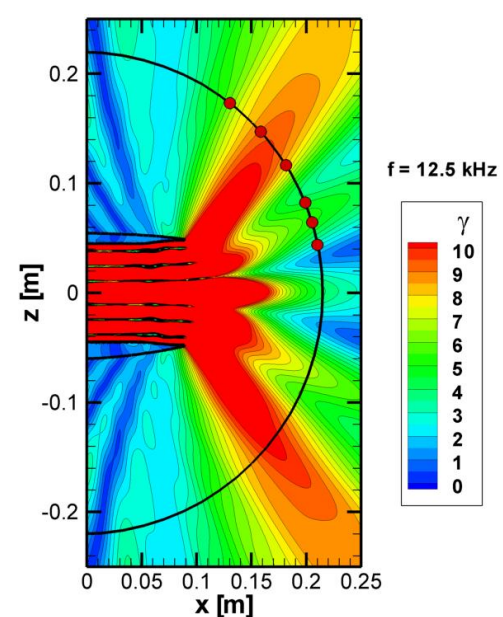

(a) $f=12.5 \mathrm{kHz}$

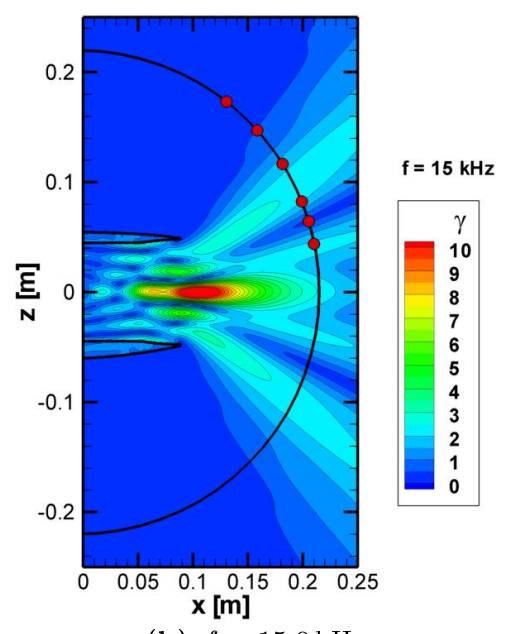

(b) $f=15.0 \mathrm{kHz}$

Fig. 17: Shielding factor contour plots, simulation results. 


\section{Assessment of Noise Shielding by Wings and Aircraft Configurations}

\subsection{Experiment 1: NACA 0012 2D Wing in AWB [17]}

The experimental setup in the AWB is shown in figure 18. The $2 \mathrm{D}$ wing with NACA 0012 profile $(c=0.2 \mathrm{~m})$ is mounted vertically in the test section with a $0.2 \mathrm{~m}$ shift away from the tunnel centerline. This is done to provide enough room for the in-flow microphone, (C) in figure 18, to be placed in the geometric far-field of the model. As can be seen in figure 18 , the whole laser was tilted $3^{\circ}$ towards the model, to avoid collision of the optical components with the wing's support when moving the source. Measurements are performed for three geometrical angles of attack, $\alpha_{g}=0^{\circ}, \pm 10^{\circ}$, corresponding to effective angles of attack, $\alpha=0^{\circ}, \pm 6^{\circ}$. The correspondence was established through comparison of the experimental pressure distributions to those obtained by CFD. The transition location was determined through stethoscope inspections, and found to occur at $\approx 0.8 c$ for $\alpha_{g}=0^{\circ}$ and $\approx 0.9 c$ and at $\approx 0.15 c$ on the suction and pressure sides respectively for $\alpha_{g}= \pm 10^{\circ}$. A $0.4 \mathrm{~mm}$ thick straight rectangular trip strip was applied at $x / c=0.6$ on the suction side to prevent the occurrence of laminar separation.

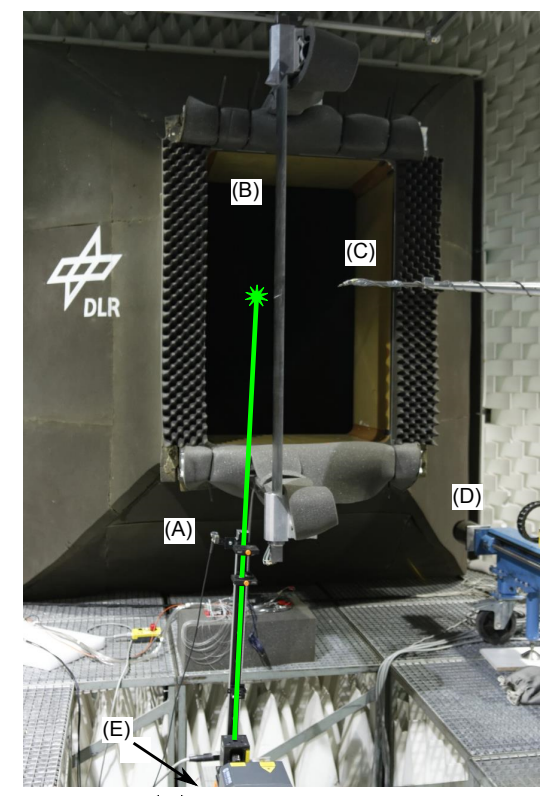

(a) Complete setup.

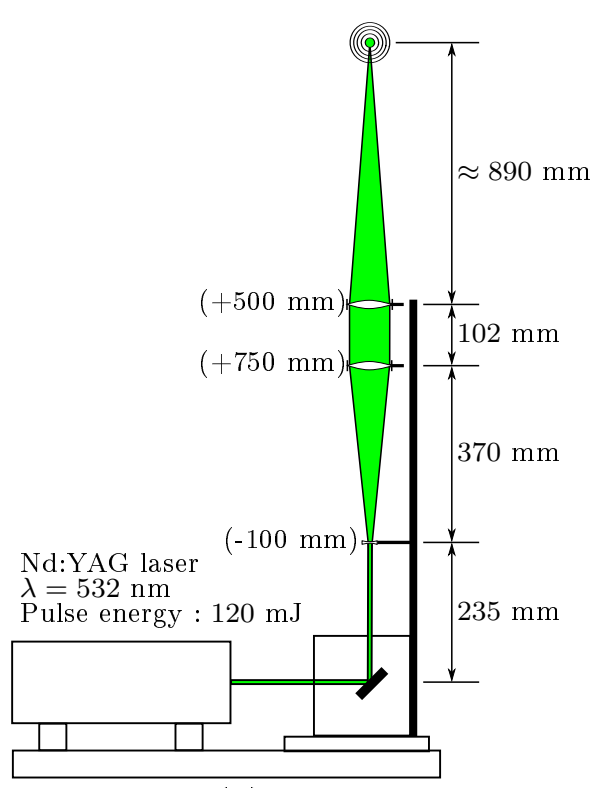

(b) Laser setup.

Fig. 18: Generic shielding setup in AWB. (a) Complete test setup : (A) Laser sound source, (B) NACA 0012 2D wing, (C) 1/8" GRAS 40DP microphone, (D) microphone positioning system, (E) laser sound source positioning system. (b) Details of the lens and laser setup.

In-flow measurements were done on linear microphone traverses, 2 chord $(0.4 \mathrm{~m})$ away from the model chord line, on the opposite side to the sound source. The in-flow 
microphone was traversed in streamwise and spanwise directions, focus is put here, however, on measurements along a streamwise microphone traverse. Also, the sound source is located at a constant distance of $25 \mathrm{~mm}$ away from the model surface. The evaluation of the shielding impact of the model requires the acquisition of data once with the model installed and once in the empty test section.

The wind tunnel nozzle was covered with acoustic foam as a preventive measure to ensure that sound wave reflections do not contaminate the measurements, especially in cases where the in-flow microphone is positioned upstream of the wing's LE. Preliminary tests revealed, however, that acoustic treatment of the ground, nozzle and positioning elements was not necessary. Reflections generally don't play an important role when using the laser sound source, due to the very short duration of the generated pressure pulses $(\approx 0.1 \mathrm{~ms})$. When a shielding object is present between the sound source and a remote receiver, one as to be more careful, however, to make sure that only the meaningful part of the measured signal is kept for further processing. This is due to the longer propagation paths of the sound waves about the shielding object, requiring longer time series to be inspected in post-processing to capture all of the relevant sound intensity.

A comparison between experimental and simulation results for selected cases is given in figure 19. In figure 19, the normalized octave band shielding level is plotted vs. microphone position below the wing for three octave bands with center frequencies; $f_{c}=7 \mathrm{kHz}, 14 \mathrm{kHz}, 28 \mathrm{kHz}$. The normalized octave band shielding levels are defined as

$$
\gamma_{p(1 / 1)}^{n}\left(f_{c}\right)=20 \log \eta_{(1 / 1)}^{n},
$$

with $\eta_{(1 / 1)}^{n}$ the octave band average of the shielding factor in each of the above frequency bands,

$$
\eta_{(1 / 1)}^{n}\left(f_{c}\right)=\left(\frac{\sqrt{2}}{f_{c}} \int_{f_{c} \sqrt{2}}^{f_{c} \sqrt{2}} \eta^{2}(f) d f\right)^{1 / 2}
$$




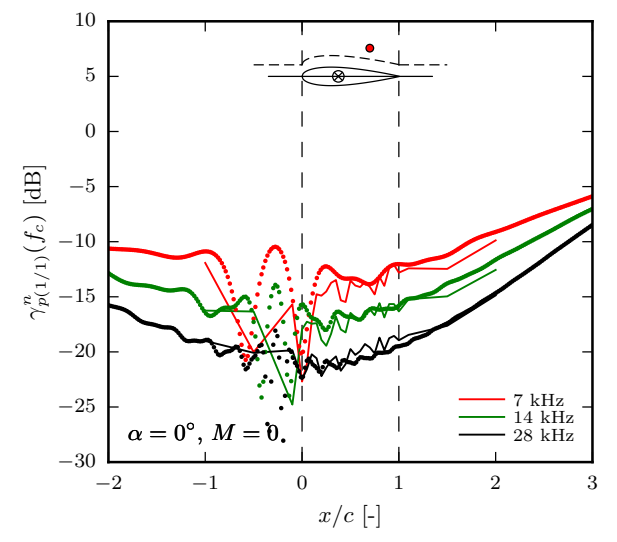

(a) $x_{s} / c=0.7, M=0.0$

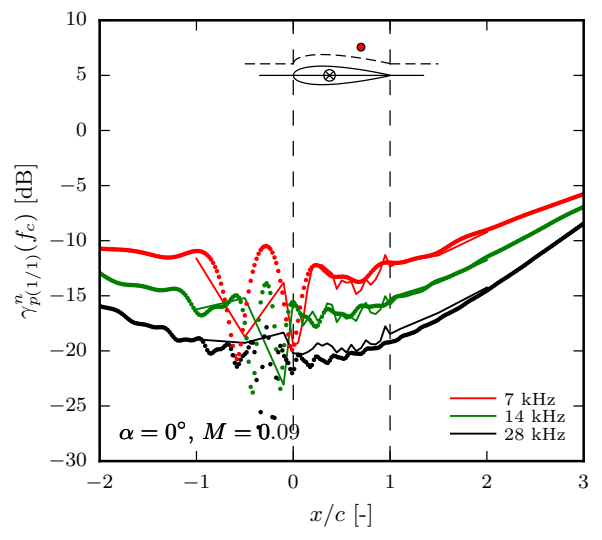

(b) $x_{s} / c=0.7, M=0.09$

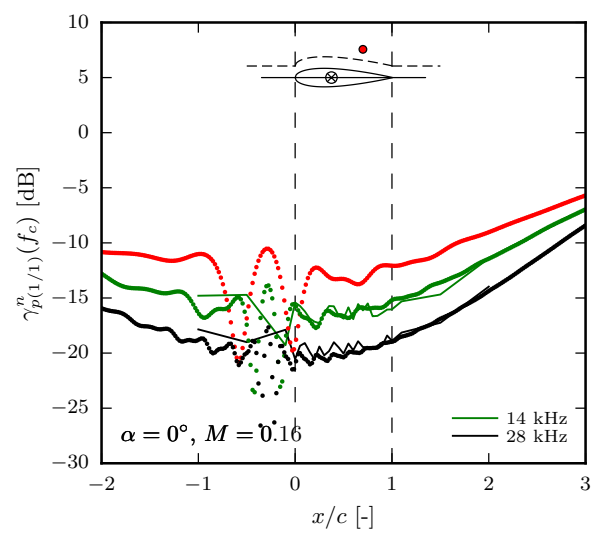

(c) $x_{s} / c=0.7, M=0.16$

Fig. 19: Normalized octave band shielding level $\left(\gamma_{p(1 / 1)}^{n}\right)$ vs. microphone position $(x / c)$ below the wing. Experimental vs. simulation results. $\alpha=0^{\circ}$, (a) $M=0$, (b) $M=0.09$ (c) $M=0.16$. In (c), data are only available for $f_{c}=14 \mathrm{kHz}$ and $28 \mathrm{kHz}$. 
4.2 Experiment 2: Hybrid Wing Body Configuration in DNW-NWB [19]

The latest set of experiments done using the laser-based methodology was concerned with the quantification of noise attenuation by 3D unconventional aircraft configurations [19]. The study was done in the DNW-NWB Low-Speed Acoustic Wind Tunnel in Braunschweig. An overview of the experimental setup is provided in figure 20. The objective here is on the one hand to generate a quality experimental data set for the validation of the shielding simulations. On the other hand the various configuration changes investigated give indications on which geometric elements influence the shielding property of this aircraft configuration most.

Figure 21 gives a comparison of the measured and calculated acoustic attenuation for the HWB configuration at $28 \mathrm{kHz}$ for four source positions (P1 to P4). The upper and centre row respectively show the measured and computed attenuation maps as directly generated from the sensor positions selected in the experiment. The global distribution of the attenuation, as simulated, corresponds quantitatively well with the measurements. However, local deviations in the distribution may be seen. The reason for these may be inferred from the spatially resolved attenuation map, depicted as a repetition from the computation results section in the bottom row of figure 21. Small deviations of the sensor positions from their nominal values may let it pick up a local maximum inside of a narrow interference fringe pattern, while on the desired position there may just be a local minimum.

For the HWB geometry, the DLR Fast-Multipole code FMCAS was used [37]. Like BEMPAR, FMCAS solves the exterior Helmholtz problem. Again, the surface of the scattering geometry is approximated by plane triangles. The surface triangulation was generated using the Gmsh code [36]. For the $7 \mathrm{kHz}$ case a surface mesh with 36500 triangles was used and for the $28 \mathrm{kHz}$ case a mesh with 555000 triangles. The memory requirements for the $7 \mathrm{kHz}$ case were about $2.5 \mathrm{~GB}$ and for the $28 \mathrm{kHz}$ case about 28 GB. 


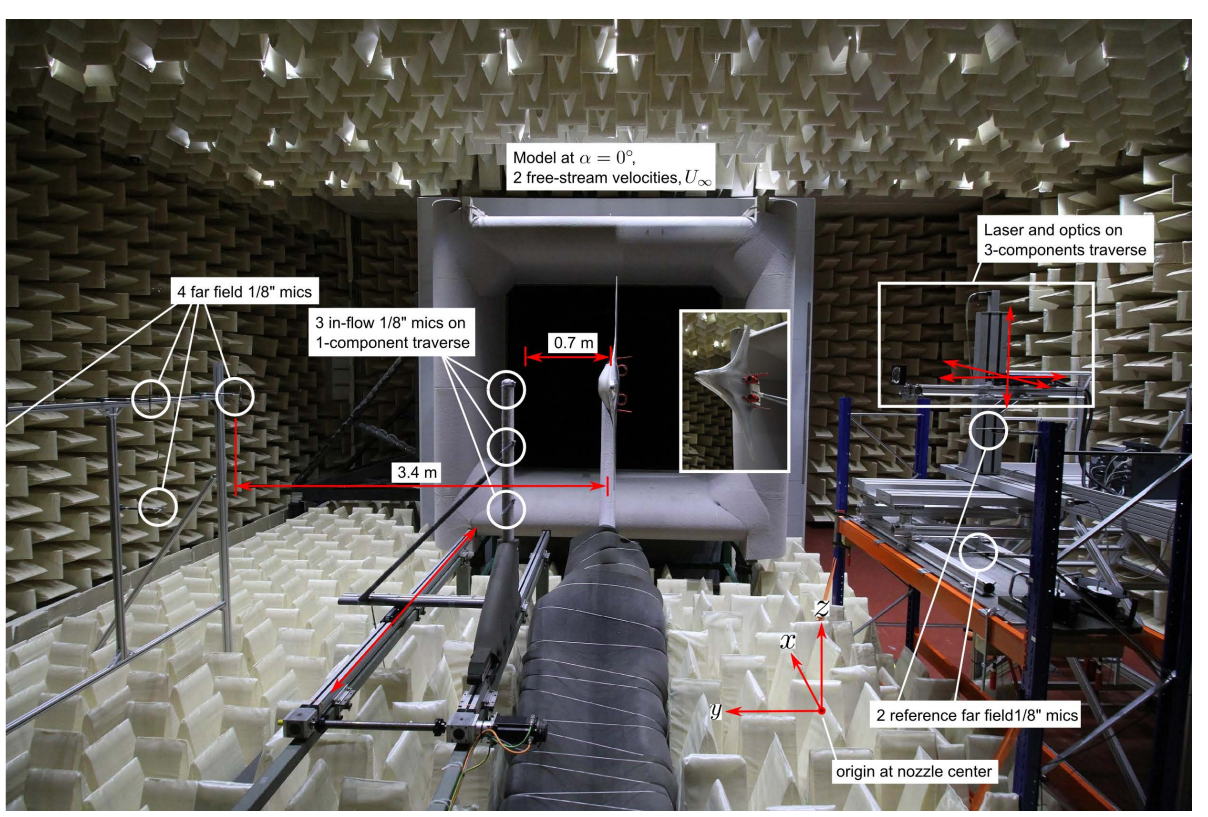

Fig. 20: Overview of the complete test setup. LNA model installed. 

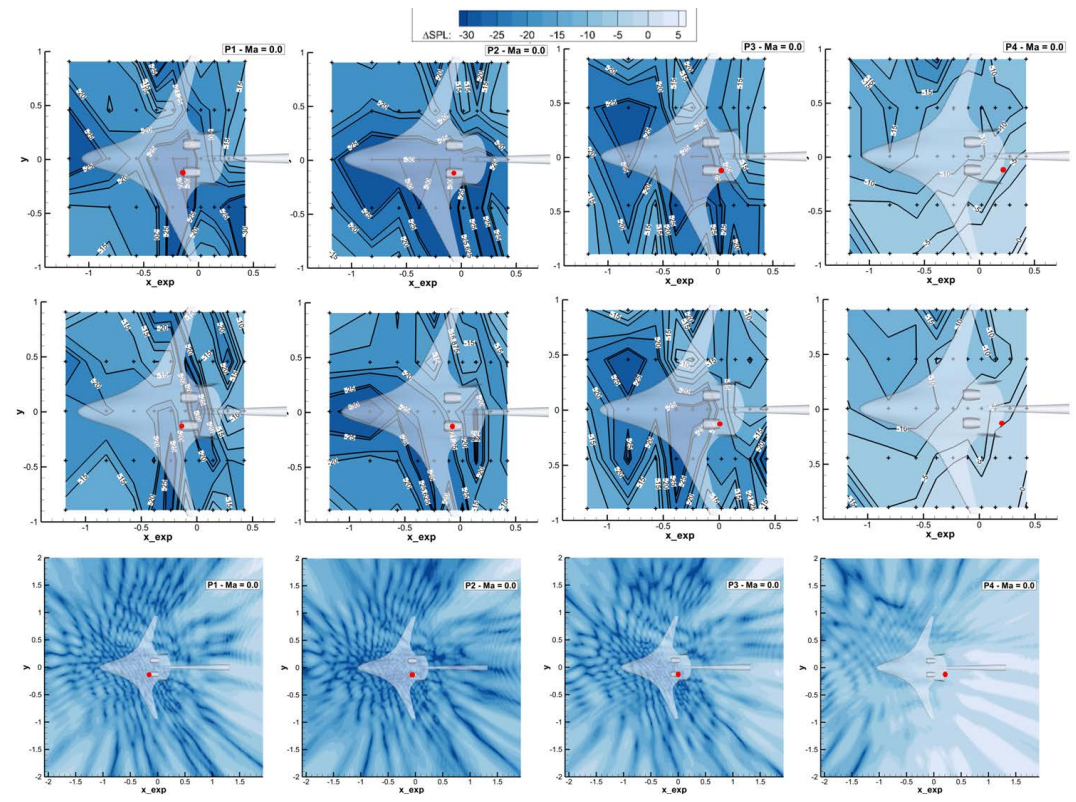

(a) $f=7 \mathrm{kHz}$
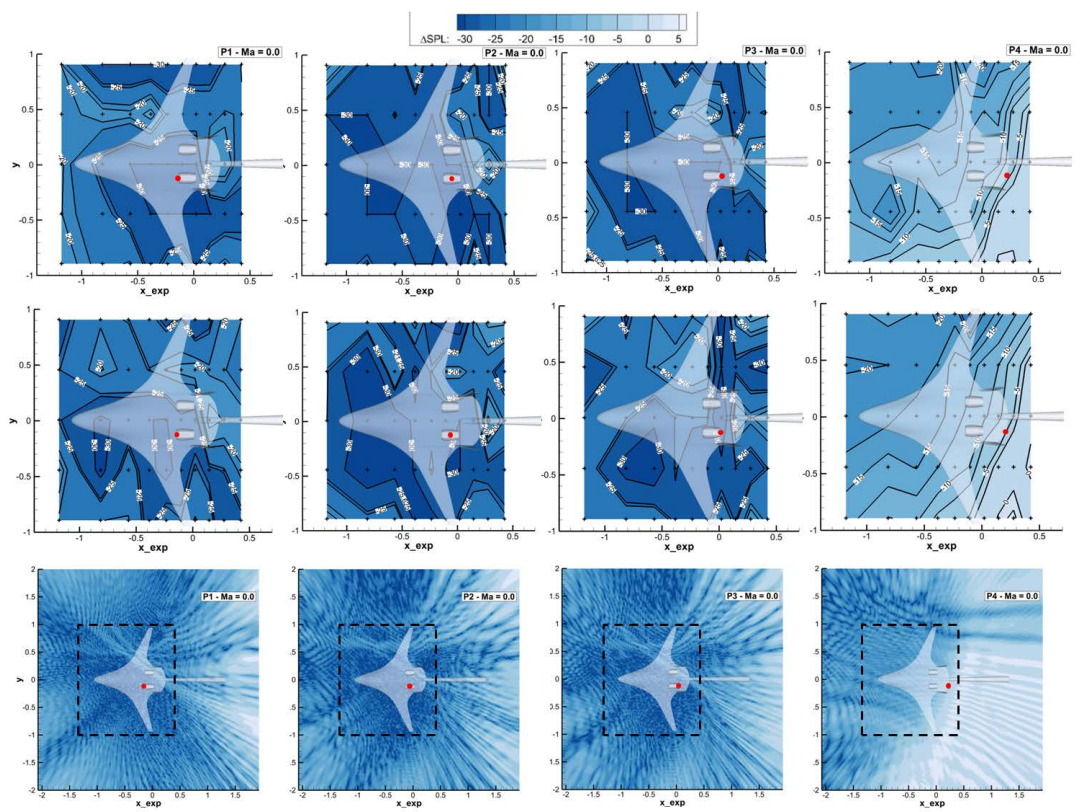

(b) $f=28 \mathrm{kHz}$

Fig. 21: Comparison of attenuation in plane $0.7 \mathrm{~m}$ below the aircraft (HWB) at $7 \mathrm{kHz}$ and $28 \mathrm{kHz}, \mathrm{M}=0$ vs. streamwise source position above the aircraft. Top row: experiment, central row: computation result at the same sampling points as in the experiment, bottom row: densely spaced sampling point 


\section{Conclusions}

A review of the most recent available experimental work on the shielding of turbomachinery noise is presented. The topic has gain a lot of attention in the last decade where an increasing number of experimental work as been published. This newly found interest is strongly motivated by recent system noise analyses emphasizing the central role of turbomachinerery noise shielding in designing the next-generation low-noise aircraft.

Early experimental work on the topic provided first indications on the noise reduction potential of turbomachinery noise achievable through shielding. Most recent experimental investigations, with focus on the hybrid wing body concept, provide detailed high-quality data covering a wide range of parametric configuration changes as well as flow related effects on the shielding effectiveness. Together, these databases enable an estimation of the noise benefit of specific configuration changes on the shielding characteristic of a low-noise aircraft design of the hybrid-wing-body type.

More detailed analysis on noise shielding will require the use of reliable numerical simulation tools. In particular, for system noise analyses, methods with quick turnaround time are required which still deliver precise and reliable data. To reach this goal, high-quality validation data are needed. Results are presented from investigations done using a broadband non-intrusive laser-based reference sound source. The methodology is shown to be appropriate for the production of high-quality experimental data. Furthermore, an analytical description of the source can be derived from a solution to the convected wave equation, making a numerical replication of the wind tunnel tests with the laser sound source possible.

\section{References}

1. Thomas, R. H., Burley, C. L., and Olson, E. D., "Hybrid wing body aircraft system noise assessment with propulsion airframe aeroacoustic experiments," International Journal of Aeroacoustics, Vol. 11, No. 3-4, 2012, pp. 369-409.

2. Guo, Y., Burley, C. L., and Thomas, R. H., "On noise assessment for blended wing body aircraft," AIAA Paper, Vol. 365, 2014.

3. Reshotko, M., Olsen, W. A., and Dorsch, R. G., "Preliminary noise tests of the engineover-the-wing concept. 2: $10 \mathrm{deg}-20 \mathrm{deg}$ flap position," 1972.

4. Dorsch, R. G., Reshotko, M., and Olsen, W. A., "Flap noise measurements for STOL configurations using external upper surface blowing," 1972.

5. Reshotko, M., Goodykoontz, J. H., and Dorsch, R. G., "Engine-over-the-wing noise research," 1973.

6. Hellstrom, G., "Noise Shielding Aircraft Configurations, A Comparison Between Predicted and Experimental Results," Ninth Congress of the International Council of Aeronautical Sciences, 1974, pp. 74-58.

7. Jeffery, R. and Holbeche, T., "Experimental studies of noise-shielding effects for a deltawinged aircraft," AIA A paper, Vol. 75, 1975.

8. Broadbent, E., Holbeche, T. A., and Butler, G. F., "Interaction of a Vortex Core with Acoustic Radiation," Euromech 34 on Control and Feedback Mechanisms in Flow Noise, Göttingen, September 1972.

9. Clark, L. R. and Gerhold, C. H., "Inlet noise reduction by shielding for the blended wing body airplane," AIAA paper, , No. 99-1937, 1999, pp. 10-12.

10. Gerhold, C. H. and Clark, L. R., "Database of inlet and exhaust noise shielding for wedgeshaped airframe," Tech. rep., 2001.

11. Gerhold, C. H., Clark, L. R., Dunn, M. H., and Tweed, J., "Investigation of acoustical shielding by a wedge-shaped airframe," Journal of sound and vibration, Vol. 294, No. 1, 2006, pp. 49-63. 
12. Ricouard, J., Davy, R., Loheac, P., Moore, A., and Piccin, O., "Rosas wind tunnel test campaign dedicated to unconventional aircraft concepts study." 10th AIAA/CEAS Aeroacoustics Conference Manchester, UK, 2004.

13. Papamoschou, D. and Mayoral, S., "Experiments on shielding of jet noise by airframe surfaces," 15th AIAA/CEAS Aeroacoustics Conference, AIAA-2009-3326, Miami, Florida, USA, 2009.

14. Czech, M. J., Thomas, R. H., and Elkoby, R., "Propulsion airframe aeroacoustic integration effects for a hybrid wing body aircraft configuration," International Journal of Aeroacoustics, Vol. 11, No. 3-4, 2012, pp. 335-367.

15. Hutcheson, F. V., Brooks, T. F., Burley, C. L., Bahr, C. J., Stead, D. J., and Pope, D. S., "Shielding of Turbomachinery Broadband Noise from a Hybrid wing Body Aircraft Configuration," 22nd AIAA/CEAS Aeroacoustics Conference, AIAA 2016-2708, Lyon, France, 2016.

16. Rossignol, K.-S., Lummer, M., and Delfs, J., "Validation of DLR's sound shielding prediction tool using a novel sound source," 11th AIA A/CEAS aeroacoustics conference, AIAA 2009-3329, Miami, USA, 2009.

17. Rossignol, K.-S. and Delfs, J., "Analysis of the Noise Shielding Characteristics of a NACA0012 2D Wing," 22nd AIAA/CEAS Aeroacoustics Conference, AIAA 2016-2795, Lyon, France, 2016.

18. Yin, J., Rossignol, K.-S., and Bulté, J., "Acoustic Scattering Experiments on Spheres For Studying Helicopter Noise Scattering," Proceedings of the 42nd European Rotorcraft Forum, Lille, France, 2016.

19. Rossignol, K.-S., Pott-Pollenske, M., Delfs, J., Silbermann, J., and Pereira Gomes, J. M., "Investigating Noise Shielding by Unconventional Aircraft Configurations," 23rd AIAA/CEAS Aeroacoustics Conference, AIAA 2017-3195, Denver, USA, 2017.

20. Rossignol, K.-S., Delfs, J., and Boden, F., "On the Relevance of Convection Effects for a Laser-Generated Sound Source," 21st AIAA/CEAS Aeroacoustics Conference, AIAA 2015-3146, Dallas, USA, 2015.

21. Hosoya, N., Nagata, M., and Kajiwara, I., "Acoustic testing in a very small space based on a point sound source generated by laser-induced breakdown: Stabilization of plasma formation," Journal of Sound and Vibration, Vol. 332, No. 19, 2013, pp. 4572-4583.

22. Radziemski, L. and Cremers, D., Laser induced plasmas and applications, Marcel Dekker Inc., New-York, June 1989.

23. Phuoc, T. X. and White, C. M., "Experimental studies of the absorption and emissions from laser-induced spark in combustible gases," Optics communications, Vol. 181, No. 4, 2000, pp. 353-359.

24. Chen, Y.-L., Lewis, J., and Parigger, C., "Spatial and temporal profiles of pulsed laserinduced air plasma emissions," Journal of quantitative spectroscopy and radiative transfer, Vol. 67, No. 2, 2000, pp. 91-103.

25. Ostrovskaya, G. and Zaŭdel', A., "Laser spark in gases," Physics-Uspekhi, Vol. 16, No. 6, 1974, pp. 834-855.

26. Villagrán-Muniz, M., Sobral, H., and Navarro-González, R., "Shock and thermal wave study of laser-induced plasmas in air by the probe beam deflection technique," Measurement Science and Technology, Vol. 14, No. 5, 2003, pp. 614.

27. Boden, F. and Delfs, J., "Development of a laser-based sound source," Inter-Noise, Honolulu, Hawaii; USA, 2006.

28. Phuoc, T. X., "Laser spark ignition: experimental determination of laser-induced breakdown thresholds of combustion gases," Optics Communications, Vol. 175, No. 4, 2000, pp. 419-423.

29. Saleh, B. and Teich, M., Fundamental of photonics, chap. 3, John Wiley \& Sons, New York, 1991, pp. 80-107.

30. Delfs, J. W., Ewert, R., Bauer, M., and Dierke, J., Numerical Simulation of Aerodynamic Noise with PIANO, Institute of Aerodynamics and Flow Technology, Department of Technical Acoustics, DLR, Lilienthalplatz 7, 38108 Braunschweig, Germany, 92015.

31. Schwamborn, D., Gerhold, T., and Heinrich, R., "The DLR TAU-Code: Recent Applications in Research and Industry," European Conference on Computational Fluid Dynamics, TU Delft, 2006.

32. Delfs, J. and Ewert, R., "Numerical Simulation of Aerodynamic Noise with PIANO." Tech. rep., DLR, Braunschweig, 2015.

33. Burton, A. J. and Miller, G. F., "The Application of Integral Equation Methods to the Numerical Solution of some Exterior Boundary Value Problems," Proceedings of the Royal Society, London, Vol. A 323, 1971, pp. 201-210. 
34. Kirkup, S. M., "BEMHELM: BEM for Helmholtz Problems," http://www.boundaryelement-method.com/helmholtz/manual/index.htm, 1998.

35. A. J. Burton and G. F. Miller. The application of integral equation methods to the numerical solution of some exterior boundary value problems. Proceedings of the Royal Society, London, A 323:201-210, 1971.

36. Christophe Geuzaine and Jean-François Remacle. Gmsh: A 3-d finite element mesh generator with built-in pre-and post-processing facilities. International Journal for Numerical Methods in Engineering, 79(11):1309-1331, 2009.

37. Markus Lummer. Installation effects - numerical investigation. In Aircraft Noise Generation and Assessment. CEAS Aeronautical Journal, submitted.

38. Joachim Schöberl. NETGEN - automatic mesh generator. Johannes Kepler University Linz, Austria. http://www.hpfem.jku.at/netgen. 